\title{
Population viability analysis and conservation of Chinese Grouse Bonasa sewerzowi in Lianhuashan Nature Reserve, north-west China
}

\author{
NAN LU and YUE-HUA SUN
}

\section{Summary}

Chinese Grouse Bonasa sewerzowi is threatened by human activity, especially during the breeding season, in the Lianhuashan Mountains, Gansu Province, north-western China. We conducted a series of simulations on the viability of this population using the computer program VORTEX. The simulations suggested that the population had an extinction probability of $17 \%$ in 100 years using data gathered from current field work. Sensitivity analysis revealed that the predicted population trend was most sensitive to chick mortality, offspring per female per year, and adult male mortality. The first two parameters are correlated with human activity such as nest loss due to egg collecting by local people. When we set initial population size to the same size as carrying capacity, 2,500 individuals would constitute a minimum viable population (MVP). This would require a forest area of about 3,780 ha, which is smaller than the size of the Lianhuashan reserve, but the current population does not constitute an MVP due to the small initial population size. Furthermore, we found that if chick mortality declined by $5 \%$ or the number of offspring produced per female increased by $5 \%$ (i.e. reducing nest loss) under the current situation, local reserve size and current population would constitute an MVP. Therefore, the most practical and simple conservation management tool would be to increase the breeding success of Chinese Grouse, especially by limiting human activity during the incubation period.

\section{Introduction}

Population viability analysis (PVA) is the quantitative evaluation of all factors and their interactions that act on populations and contribute to the risks of short-term and long-term decline or extinction (Boyce 1992). Challenges in conservation biology include the identification and measurement of risks to endangered species and the evaluation of actions to reduce these risks (Drechsler and Burgman 2004). PVA attempts to assess the likelihood of future events based on currently available data and theory (Shaffer 1990). It is a useful and increasingly popular tool (Engen and Sæther 2000) and has been shown to provide reasonable predictions of extinction risk for well-studied species (i.e. it requires adequate life-history information) (Brook et al. 2000, McCarthy and Broome 2000), or even to inform policy decisions (Beissinger 2002). PVA enables wildlife managers to forecast the fate of populations, allowing the development of pre-emptive management actions and strategic research programmes (Clark et al. 1991, Lindenmayer et al. 1993). Lacy (1993) developed a generic and individual-based population simulation model VORTEX to assess extinction probability by simulating the population dynamic process. The program was written originally to model mammalian and avian populations (Miller and Lacy 2003), and has been applied widely to endangered species conservation (Marmontel et al. 1991, Bustamante 1996). 
The minimum viable population (MVP) is an output of PVA and is equivalent to the smallest size required for a population or species to have a predetermined probability of persistence for a given length of time (Shaffer 1981). MVP size implies that there is some threshold for the number of individuals ensuring that a population will persist for a given interval of time at some acceptable level of risk (Rai 2003). This can be useful in conservation management, such as reserve construction or species recovery (Clark et al. 1991). The minimum area requirement (MAR) is the area of suitable habitat required to support the MVP (Shaffer 1987).

Here we conducted a PVA for a population of Chinese Grouse Bonasa sewerzowi, a species threatened by illegal hunting, egg collecting, and also by habitat loss and its restricted geographical distribution (Storch 2000). This grouse is endemic to China and inhabits isolated remnants of the coniferous mountain forests of Gansu, Qinghai, Sichuan, Yunnan and Tibet (Sun 2000). It is the southernmost, and one of the rarest, grouse species in the world (Storch 2000, Sun et al. 2003). In Lianhuashan Nature Reserve (LNR), where we conducted our study, management has not previously taken the future survival of the Chinese Grouse population into account and it was unknown whether the LNR was large enough to support a viable population.

We conducted simulations using VORTEX software to: (I) predict the survival probability of this Chinese Grouse population; (2) identify the factors affecting this population's viability; (3) evaluate the MVP and MAR by simulating the population dynamics of the population, and (4) assess the population's current status.

\section{Methods}

\section{Study area and local Chinese Grouse population}

LNR is located at $34^{\circ} 45^{\prime}-35^{\circ} 06^{\prime} \mathrm{N}, 103^{\circ} 27^{\prime}-103^{\circ} 51^{\prime} \mathrm{W}$, in southern Gansu. The reserve was established in 1983, and was gazetted as a national reserve in 2003. Many endemic birds occur in the coniferous forests such as Blood Pheasant Ithaginis cruentus and Snowy-cheeked Laughingthrush Garrulax sukatschewi. The reserve is linked to the Yeliguan Forestry Farm (where the other local grouse population occurs) only by a forest corridor less than $\mathrm{I} \mathrm{km}$ wide. However, as reported by Sun et al. (2006), no grouse has been found in this corridor because the habitat is locally fragmented, due to natural aridity, and the effects of agriculture and deforestation (Zheng and Wang 1998). Thus, the LNR Chinese Grouse population was considered to be isolated.

\section{Parameter values and analysis}

The Chinese Grouse has been studied in LNR for about 15 years (Swenson et al. 1996, Larsson et al. 2003, Sun et al. 2003). This research provides some background on the species and, more importantly, forms the basis for our simulation input. The software package VORTEX 9.72 (Lacy et al. 2003) was used for the analysis. The probability of population persistence was estimated by running the simulation 100 times to simulate 100 years. We defined extinction to have occurred at the point when individuals of only one sex remained. We predicted the future trend of this population, and evaluated the adequacy of current management; the main input parameter values are shown in Table 1.

The age-specific mortality rates and reproductive data were gathered from 73 radio-marked Chinese Grouse followed from 1995 to 2000 (Sun et al. 2003). VORTEX defines breeding as the time when the first offspring are born, not the age of onset of sexual maturity or the age of first conception (Miller and Lacy 2003). Males could potentially participate in reproduction in the first breeding season after they were born, but this has not yet been documented (Y.-H. Sun unpubl. data), so the initial male breeding age was set at two years. Zhang and Zheng (2007) used maximum reproductive age derived from captive individuals. However, birds in their natural habitats are highly unlikely to achieve the maximum physiological reproductive age, because of predation and other mortality factors. Here the maximum age of reproduction was set 
Table 1. Summary of initial parameters used for population viability analysis of the Chinese Grouse in LNNR, Gansu Province, China.

\begin{tabular}{ll}
\hline Parameters & Value \\
\hline Mating system & Monogamy \\
Female initial breeding age & 1 \\
Male initial breeding age & 2 \\
Sex ratio (male/[male+female]) & 0.64 \\
Sex ratio at birth & 0.5 \\
Number of offspring per female per year & $3.62(\mathrm{SD}=0.41)$ \\
Maximum age of reproduction & 7 \\
Males in breeding pool & $56 \%$ \\
Female mortality at age o-1 & $0.68(\mathrm{EV}=0.09 \mathrm{SD})$ \\
Female mortality after age 1 & $0.39(\mathrm{EV}=0.07 \mathrm{SD})$ \\
Male mortality at age o-1 & $0.68(\mathrm{EV}=0.09 \mathrm{SD})$ \\
Male mortality at age $1-2$ & $0.34(\mathrm{EV}=0.08$ SD) \\
Male mortality after age 2 & $0.34(\mathrm{EV}=0.08 \mathrm{SD})$ \\
Carrying capacity $(K)$ & $2,695(\mathrm{EV}=0.00 \mathrm{SD})$ \\
Initial population size & 700 \\
Harvest or supplementation? & No \\
\hline
\end{tabular}

as the same as average life expectancy - seven years (Y.-H. Sun unpubl. data). Chinese Grouse is strictly monogamous according to field observations (Wang 1991, Liu and Geng 1994), and all adult males and females were assumed to have the same opportunity to breed. Additionally, we started to tally offspring at hatching, following Miller and Lacy (2003) rather than using clutch size; thus mortality after the chicks hatched should be used as the mortality from age o to 1 .

Sun et al. (2003) reported chick mortality for the first 13 weeks after hatching, and we arbitrarily used the mortality of chicks during this period, which was collected from 32 broods between 1995 and 1998 due to the lack of mortality data for the whole first year. As all grouse found during the breeding season were confined to forests (Sun et al. 2006), we calculated the initial population size and carrying capacity using the local forest area of 4,075 ha derived from Song and Wang (2000). The mean territory size of paired male Chinese Grouse in spring is about 2.10 ha, and of unpaired about 2.70 ha (Sun 2004), so we can derive a carrying capacity of about 2,695 (details in Appendix 2). The population density of Chinese Grouse in spring averaged 17.2 $100 \mathrm{ha}^{-1}$ (Sun et al. 2003) so the initial population size was estimated to be $700(17.2 \times 40.75)$. As the sex ratio has been calculated as $64 \%$ male and $36 \%$ female (Sun et al. 2003), this resulted in an estimated 448 males and 252 females. We divided this initial population into 224 males and 126 females of age 1 and 224 males and 126 females of age 2, due to lack of more detailed field data.

\section{Sensitivity analysis}

Since not all parameters included in the PVA model are likely to be equally important in influencing the population's long-term viability (Miller and Lacy 2003, Zhang and Zheng 2007), we assessed the sensitivity of the model's response when we varied the selective parameters at the same level. Sensitivity was calculated as follows: $\mathrm{Sx}=(\Delta \mathrm{x} / \mathrm{x}) /(\Delta \mathrm{P} / \mathrm{P})$, where $\Delta \mathrm{x} / \mathrm{x}$ is the change in population size resulting from a change of $\Delta \mathrm{P} / \mathrm{P}$ in the parameter $\mathrm{P}$ (Pulliam et al. 1992). Here the level of $\Delta \mathrm{P}$ was set at $5 \%$ for the assessment, and we selected chick mortality from age o to 1 (CM); adult female mortality after age I (AFM); subadult male mortality from age 1 to 2 (SMM); adult male mortality after age 2 (AMM); carrying capacity $(K)$, and offspring per female per year $(\mathrm{R})$. After we identified the most sensitive parameters, they would be set at different levels $(+10 \%$, $+5 \%,-5 \%$ and $-10 \%)$ to determine the effects on the population. An additional simulation on inbreeding was carried out to establish the sensitivity of that model. 


\section{MVP and MAR}

Until now, there has been no universally acknowledged standard level at which to define viable populations (Shaffer 1981, Shaffer 1987, Boyce 1992). Typically, $<1 \%$ or $5 \%$ probability of extinction over 100 years has been considered as the thresholds determining viability (Grimm and Storch 2000). For this study, we used a $5 \%$ threshold to determine the MVP. The IP and $K$ were initially set at 1,00o and increased step by step until the extinction rate was $<5 \%$. The MAR was calculated by $2.1 \mathrm{~m}+$ $2.7 \mathrm{n}$ according the equations see in Appendix 2, while MVP $=\mathrm{m}+\mathrm{n}$.

\section{Results}

Predicting the survival of the study population

According to the results of the simulation, this population would have a $17 \%$ probability of extinction in 100 years (Figure 1 ). The mean stochastic growth rate $(r)$ was -0.0163 , which indicated a declining trend, but the population increased for the first five years (Figure 2). The mean final population size for the extant cases was $643 \pm 87$ SE (340 males and 303 females) with a mean expected heterozygosity of 0.901 (0.013 SE) and an inbreeding coefficient of 0.078 (0.011 SE). For the simulated populations that became extinct, the mean time to first extinction was $80.12(4.58 \mathrm{SE})$ years.

\section{Sensitivity analysis}

Model output was most sensitive to CM $\left(S_{x}=18.26\right), R(S x=14.19)$, and AMM ( $\left.S x=11.63\right)$ (Figure 3). By manipulating these three parameters, we found that if $\mathrm{CM}$ declined, or R increased by $5 \%$, this population would have a positive rate of growth $(r=0.0192$ and 0.0018 , respectively), and extinction rates were calculated to be $1 \%$ and $4 \%$, respectively. The third most sensitive parameter, AMM, had to be decreased by 10\% to result in a positive $r$ (o.0ogo; Table 2). Furthermore, if inbreeding depression were removed, the extinction probability was decreased to $9 \%$, but heterozygosity did not change much (Table 2 ).

\section{The assessment of MVP and MAR}

With the IP set equal to $K$, a population containing 2,500 individuals had a predicted extinction rate $<5 \%$ and so would constitute an MVP (Appendix 1). Due to the sex ratio of o.64 (Table 1 ),

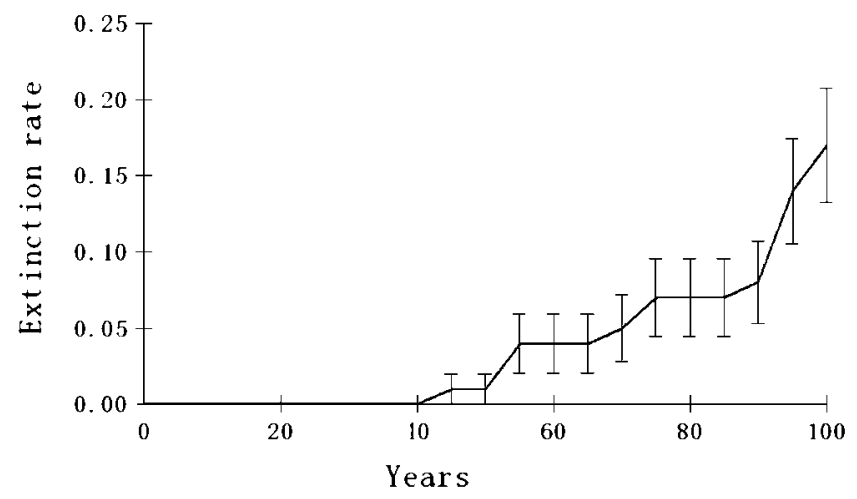

Figure 1. The simulated extinction probabilities of Chinese Grouse populations in Lianhuashan Nature Reserve, Gansu Province, China (with Standard Error shown by vertical bar). 


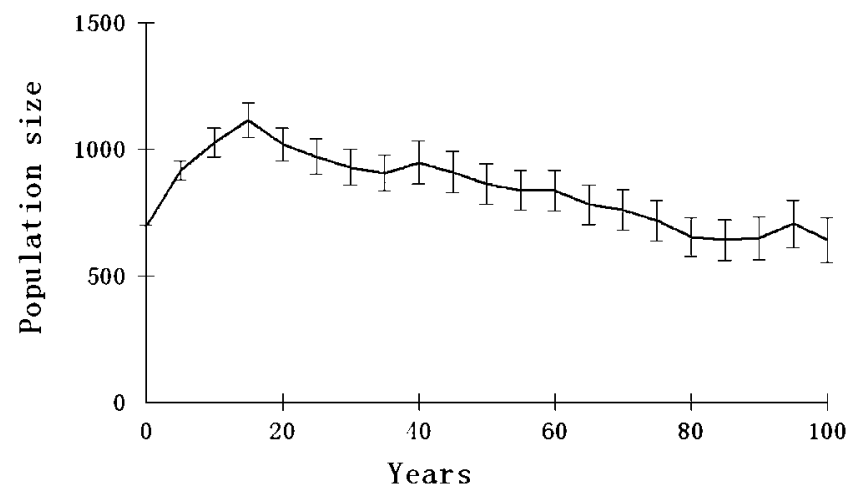

Figure 2. The simulated population development trend of Chinese Grouse during the next 100 years in Lianhuashan Nature Reserve, Gansu Province, China (with Standard Error shown by vertical bar, values were calculated from simulations not going extinct).

there would be 1,600 male individuals, and thus the relevant forest area needed to support them is about 3,780 ha, smaller than the current area of 4,075 ha.

\section{Discussion}

The risk of extinction and parameter sensitivity evaluation

PVA is a widely used tool for assessing extinction risk and guiding conservation management (Boyce 1992), but there have been concerns about its accuracy and practicality in conservation planning (Brook 2000, Brook et al. 2000, Burgman and Possingham 2000). Miller and Lacy (2003) suggested that uncertainty in simulations could occur due to limited field data, potentially causing large sampling errors. Because we lack accurate estimates of initial age-specific individual numbers, the individuals were all set at age 1 or 2 initially, which might explain the increase in the population at the beginning (Figure 2). Furthermore, we ignored effects of catastrophes, such as a high level of precipitation or extremely low temperature during the breeding season, which

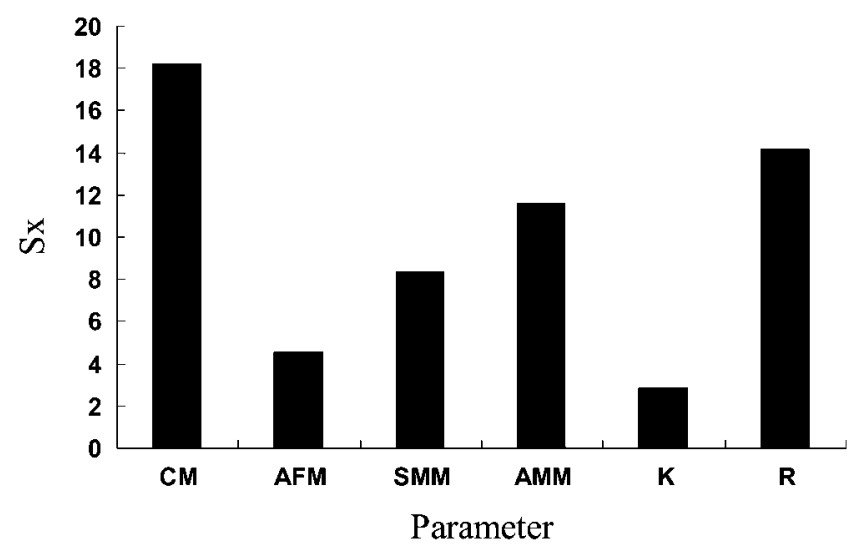

Figure 3. The sensitivity indices (Sx) of population parameters used in the simulations of the population of Chinese Grouse at Lianhuashan Nature Reserve, Gansu Province, China. 
Table 2. Sensitivity response to the change of selected parameters at different levels used in the simulations of the population of Chinese Grouse at Lianhuashan Nature Reserve, Gansu Province, China.

\begin{tabular}{|c|c|c|c|c|c|c|}
\hline Parameter level & $\begin{array}{l}\text { Rate of } \\
\text { increase (SE) }\end{array}$ & $\begin{array}{l}\text { Probability } \\
\text { of extinction } \\
\text { (SE) }\end{array}$ & $\begin{array}{l}\text { Mean time } \\
\text { to extinction } \\
\text { (SE) }\end{array}$ & $\begin{array}{l}\text { Expected } \\
\text { heterozygosity } \\
\text { (SE) }\end{array}$ & $\begin{array}{l}\text { Inbreeding } \\
\text { coefficient } \\
\text { (SE) }\end{array}$ & $\begin{array}{l}\text { Final } \\
\text { population } \\
\text { size }(\mathrm{SE})^{*}\end{array}$ \\
\hline $\begin{array}{l}\text { Current population } \\
\mathrm{CM}\end{array}$ & $-0.0163(0.0021)$ & $0.1700(0.0376)$ & $80.12\left(4.5^{8}\right)$ & 0.9008 (0.0134) & $0.0777(0.0113)$ & $642.69(87.28)$ \\
\hline$+10 \%$ & $-0.0964(0.0031)$ & 1 & $54.15(1.46)$ & 1 & / & / \\
\hline$+5 \%$ & $-0.0702(0.0028)$ & $0.8800(0.0325)$ & $65.06(1.77)$ & 0.8896 (0.0269) & $0.0764(0.0236)$ & $386.42(219.83)$ \\
\hline$-5 \%$ & 0.0192 (0.0019) & 0.0100 (0.0099) & 97.00 (o.0o) & $0.9668(0.0036)$ & $0.0309(0.0034)$ & $1,624.23(85.04)$ \\
\hline$-10 \%$ & $0.0540(0.0018)$ & $\mathrm{o}$ & I & $0.9847(0.0004)$ & 0.0149 (0.0005) & $2,357.76(43.68)$ \\
\hline \multicolumn{7}{|l|}{ AMM } \\
\hline$+10 \%$ & $-0.0464(0.0023)$ & $0.5400(0.0498)$ & $74.13(2.08)$ & $0.8176(0.0259)$ & 0.1462 (0.0269) & $137.43(33.26)$ \\
\hline$+5 \%$ & $-0.0294(0.0021)$ & $0.3000(0.0458)$ & $75.20(2.39)$ & 0.9077 (0.0090) & $0.0662(0.0087)$ & $318.90(58.28)$ \\
\hline$-5 \%$ & $-0.0032(0.0020)$ & $0.0900(0.0286)$ & $77.00(3.54)$ & $0.9444(0.0082)$ & $0.0539(0.0097)$ & $900.26(80.88)$ \\
\hline$-10 \%$ & $0.0090(0.0020)$ & $0.0700(0.0255)$ & $83.57(5.85)$ & $0.9589(0.0048)$ & $0.0328(0.0031)$ & $1,306.35(85.62)$ \\
\hline \multicolumn{7}{|l|}{$\mathrm{R}$} \\
\hline$+10 \%$ & $0.0158(0.0020)$ & o & / & $0.9689(0.0035)$ & $0.0272(0.0027)$ & $1,439.95(86.49)$ \\
\hline$+5 \%$ & $0.0018(0.0020)$ & $0.0400(0.0196)$ & $72.50(13.90)$ & $0.9326(0.0120)$ & 0.0526 (0.0104) & $1,025 \cdot 31(86.77)$ \\
\hline$-5 \%$ & $-0.0386(0.0022)$ & $0.4400(0.0496)$ & $80.43(2.31)$ & $0.8428(0.0244)$ & $0.1469(0.0290)$ & $276.59(76.47)$ \\
\hline$-10 \%$ & $-0.0593(0.0025)$ & $0.7500(0.0433)$ & $68.40(2.14)$ & $0.8625(0.0241)$ & $0.0971(0.0235)$ & $256.12(93.64)$ \\
\hline $\begin{array}{l}\text { Without } \\
\text { inbreeding }\end{array}$ & $-0.0109(0.0021)$ & $0.0900(0.0286)$ & $74.00(4.14)$ & $0.9103(0.0092)$ & $0.0779(0.0076)$ & 623.38 (80.10) \\
\hline
\end{tabular}

${ }^{*}$ Here represents the mean final population size for successful cases after 100 years. 
could have a great impact on survival of individuals or even the whole population. However, the predicted future trend of this population still showed a gradual decline and a relatively high extinction probability of $17 \%$ (Figure 2 ).

The sensitivity analysis showed that $\mathrm{CM}$ was the most influential parameter and was exceptionally high for this population, compared to the figures reported by Bergerud and Gratson (1988) for grouse in general: a mean chick mortality rate of 0.44 in 28 studies of nine grouse species, and 0.47 for forest grouse. Decreasing or increasing CM by as little as $5 \%$ has very different implications for the future security of this population; the extinction rate is predicted to change to $1 \%$ and $88 \%$, with heterozygosity of 0.9668 and 0.8896 , respectively. Through field observations, we found that the highest chick mortality occurred in the first three weeks when the chicks' feathers were not developed fully and they could not fly, thus limiting their chances of escape if detected by predators. Bergerud and Gratson (1988) proposed that thermoregulation limitations might also explain the high mortality during this period. Additionally, most of the data on chick mortality were collected from radio-tracked individuals. Sometimes we had to approach them quite closely, because their cryptic colouring was quite similar to the environment. Thus, research activity might have influenced the foraging behaviour of the chicks, and, more importantly, attracted predators (Sun et al. 2003).

The second most sensitive parameter, $\mathrm{R}$, was only 3.62 for Chinese Grouse, but the average clutch size was 6.07, reflecting a breeding success of only $60 \%$ due to a nest loss of $37 \%$ (Sun et al. 2003). By increasing R by $5 \%$ or $10 \%$, the extinction probability would decline from $17 \%$ to $4 \%$ and $0 \%$ respectively, and heterozygosity would also increase (Table 2). One artificial factor that affects nest success negatively is the apparently high rate of egg collecting by people, which causes 10-29\% of nest losses (Sun et al. 2003). Most local people did not know that the Chinese Grouse was an endangered species and was protected by the government, and they collected and ate the eggs whenever they found them. Furthermore, the frequency of human activities such as bamboo cutting and mushroom collecting is particularly high during the incubation period, which increases the nest encounter rate. Therefore, in order to reduce human predation on the eggs, and increase nesting success, we currently pay a relatively high reward (20 yuan) to people who find and report nests of Chinese Grouse and some other rare species, such as Blood Pheasant. Six Chinese Grouse nests have been reported since 2007 (J. Wang pers. comm.).

Miller and Lacy (2003) indicated that a relative scarcity of breeding males may limit reproduction by females in a monogamous system. Male mortality, especially AMM, was indicated to be more important than female mortality in this population, although the sex ratio was skewed with more males, and only about $56 \%$ of males in the breeding pool would have the chance to breed at present. We suggest more detailed simulations using other modelling tools should be conducted on the mortality of adult males to investigate this.

\section{MVP, MAR and conservation}

Grimm and Storch (2000) considered that MVP represented the minimum carrying capacity of a habitat needed for viability, rather than the threshold population size needed for viability under a given habitat which is large enough. Reed et al. (2003) also calculated MVP by setting the IP equal to $K$ to assess the minimum capacity. Our simulation results revealed that current reserve size could satisfy population viability in the present situation. However, the MVP was calculated under the assumption that IP is equal to $K$, which refers to an ideal state. The population size is smaller than $K$ in LNR at present (Table 1 ), probably due to heavy deforestation and hunting before the reserve was established. Sun et al. (2003) also indicated that the density estimate for this population is probably rather low due to some birds having been missed. We also noted that if $\mathrm{CM}$ was reduced by only $5 \%$ (from 0.680 to 0.646 ) or $\mathrm{R}$ increased by $5 \%$ (from 3.620 to 3.801), the current population in LNR would constitute an MVP (see Table 2). Therefore, a practical and simple method to protect the Chinese Grouse population in LNR is to improve its 
breeding success, especially by limiting human activity such as egg collecting and avoiding intensive research work during the breeding period.

Because of low household incomes and the traditional lifestyle, local people within LNR regularly collect dead wood, arrow bamboo, mushrooms and other edible plants, and even occasionally cut trees illegally in the mature forest (Sun et al. 2003). Thus, not only should we improve the management of the reserve through an ecological perspective, but also consider the social and economic conditions (Armbruster and Lande 1993). More awareness of biodiversity conservation and locally endemic and/or endangered species such as Chinese Grouse should be conducted in local villages, schools and so on. This would be much more effective than enforcement of regulations. On the other hand, finding more ways to stimulate economic development, such as developing tourism and attracting more business investment would essentially reduce human impacts during the breeding season.

\section{Acknowledgements}

The work is supported by National Natural Sciences Foundation of China (30620130110) and the Chinese Academy of Sciences. Dr. Jon E. Swenson helped us to improve the grammar, style and language of the paper. We also thank Dr. Yi Tao, two anonymous referees and the associate editor for their valuable comments.

\section{References}

Armbruster, P. and Lande, R. (1993) A population viability analysis for African Elephant (Loxodonta africana): How big should reserves be? Conserv. Biol. 7: 602610.

Beissinger, S. R. (2002) Population viability analysis: past, present, future. Pp. 5-17 in: S. R. Beissinger and D. R. McCullough, eds. Population viability analysis. Chicago, USA: University of Chicago Press.

Bergerud, A. T. and Gratson, M. W., eds. (1988) Adaptive strategies and population ecology of northern grouse. Minneapolis, USA: University of Minnesota Press.

Boyce, M. S. (1992) Population viability analysis. Annu. Rev. Ecol. Syst. 23: 481506.

Brook, B. W. (200o) Pessimistic and optimistic bias in population viability analysis. Conserv. Biol. 14: 564-566.

Brook, B. W., O'Grady, J. J., Chapman, A. P., Burgman, M. A., Akçakaya, H. R. and Frankham, R. (2000) Predictive accuracy of population viability analysis in conservation biology: Nature 404: 385-387.

Burgman, M. and Possingham, H. P. (2000) Population viability analysis for conservation: the good, the bad and the undescribed. Pp. $97^{-112}$ in A. G. Young and G. M.
Clarke, eds. Genetics, demography and viability of fragmented populations. Cambridge, UK: Cambridge University Press.

Bustamante, J. (1996) Population viability analysis of captive and released Bearded Vulture populations. Conserv. Biol. 10: 822-831.

Clark, T. W., Backhouse, G. N. and Lacy, R. C. (1991) The population viability assessment workshop: A tool for threatened species management and conservation. Endangered Species Update 8: $1-5$.

Drechsler, M. and Burgman, M. A. (2004) Combining population viability analysis with decision analysis. Biodivers. Conserv. 13: 115-39.

Engen, S. and Sæther, B. E. (2000) Predicting the time to quasi-extinction for populations far below their carrying capacity. J. Theor. Biol. 205: 649-658.

Grimm, V. and Storch, I. (200o) Minimum viable population size of capercaillie Tetrao urogallus: results from a stochastic model. Wildl. Biol: 219-225.

Lacy, R. C. (1993) Vortex: a computer simulation model for population viability analysis. Wildlife Res. 20: 45-65.

Lacy, R. C., Borbat, M. and Pollak, J. P. (2003) VORTEX: A stochastic simulation of the 
extinction process. Version 9. Brookfield, IL: Chicago Zoological Society.

Larsson, J. K., Sun, Y., Fang, Y., Segelbacher, G. and Höglund, J. (2003) Microsatellite variation in a Chinese Grouse Bonasa sewerzowi population: signs of genetic impoverishment? Wildl. Biol. 9: 261-266.

Lindenmayer, D. B., Clark, T. W., Lacy, R. C. and Thomas, V. C. (1993) Population viability analysis as a tool in wildlife conservation policy: with reference to Australia. Environ. Manage. 17: 745-758.

Liu, N. F., and Geng, Z. (1994) Reproduction of Severzov's hazel grouse (Bonasa sewerzowi). Gibier Faune Sauvage 11: 39-49.

Marmontel, M., Humphrey, S. R. and O'Shea, T. J. (1991) Population viability analysis of the Florida Manatee (Trichechus manatus latirostris), 1976-1991. Conserv. Biol. 11: 467-481.

McCarthy, M. A., and Broome, L. S. (2000) A method for validating stochastic models of population viability: a case study of the mountain pygmy-possum (Burramys parvus). J. Anim. Ecol. 69: 599-607.

Miller, P. S. and Lacy, R. C. (2003) VORTEX: A stochastic simulation of the extinction process. Version 9.21 user's manual. Apple Valley, MN: Conservation Breeding Specialist Group (SSC/IUCN).

Pulliam, H. R., Dunning, J. B. and Liu, J. (1992) Population dynamics in a complex landscape: a case study. Ecol. Applic. 2: 165-177.

Rai, U. K. (2003) Minimum sizes for viable population and conservation biology. Our Nature 1: 3-9.

Reed, D. H., O'Grady, J. J., Brook, B. W., Ballou, J. D. and Frankham, R. (2003) Estimates of minimum viable population sizes for vertebrates and factors influencing those estimates. Biol. Conserv. 113: 23-34.

Shaffer, M. L. (1981) Minimum population sizes for species conservation. BioScience 31: 131-134.

Shaffer, M. L. (1987) Minimum viable populations: coping with uncertainty. Pp. 69-
86 in M. E. Soulé, ed. Viable populations for conservation. Cambridge, UK: Cambridge University Press.

Shaffer, M. L. (1990) Population viability analysis. Conserv. Biol. 4: 39-40.

Song, C. and Wang, C. (2000) Scientific survey of the Gansu Lianhuashan Nature Reserve. Beijing, China: China Forestry Publishing House.

Storch, I. (2000) Grouse status survey and conservation action plan 2000-2004. Gland, Switzerland: WPA/BirdLife/IUCN SSC Grouse Specialist Group.

Sun, Y. (2000) Distribution and status of the Chinese Grouse. Wildlfe Biol. 6: 275-279.

Sun, Y. (2004) Distribution, reproductive strategy and population biology of the Chinese Grouse (Bonasa sewerzowi). PhD thesis, Beijing Normal University.

Sun, Y., Klaus, S., Fang, Y., Selsam, P. and Jia, C. (2006) Habitat isolation and fragmentation of the Chinese Grouse (Bonasa sewerzowi) at Lianhuashan Mountains, Gansu, China. Acta Zool. Sin. 52 (Supplement): 202-204.

Sun, Y., Swenson, J. E., Fang, Y., Klaus, S. and Scherzinger, W. (2003) Population ecology of the Chinese Grouse Bonasa sewerzowi, in a fragmented landscape. Biol. Conserv. 110: $177-184$.

Swenson, J. E., Sun, Y. and Liu, N. (1996) A potential method for age determination of the Chinese Grouse Bonasa sewerzowi. J. Ornith. 137: 255-258.

Wang, X. (1991) Chinese Grouse. In T. Lu, ed. The rare and endangered gamebirds in China. Fujian, China: Fujian Science and Technology Press.

Zhang, Y. and Zheng, G. (2007) A population viability analysis (PVA) for Cabot's Tragopan (Tragopan caboti) in Wuyanling, south-east China. Bird Conserv. Internatn. 17: 151-161.

Zheng, G. and Wang, Q. (1998) China Red Data Book of endangered animals. Aves. Beijing, China: National Environmental Protection Agency, Endangered Species Commission, P.R.C., Science Press. 
NAN LU ${ }^{\dagger}$, YUE-HUA SUN*

Key Laboratory of Animal Ecology and Conservation Biology, Institute of Zoology, Chinese Academy of Sciences, Beijing 100101, People's Republic of China

${ }^{\dagger}$ Current address: Graduate University of Chinese Academy of Sciences, Beijing 100049, People's Republic of China

*Author for correspondence; email: sunyh@ioz.ac.cn

Received 22 December 2008; revision accepted 24 September 2009;

Published online 19 March 2010

\section{Appendix 1.}

Estimates of the Minimum Viable Population sizes of Chinese Grouse population at Lianhuashan Nature Reserve, Gansu Province, China.

\begin{tabular}{lll}
\hline $\mathrm{IP} / K$ & Probability of Extinction $(\mathrm{SE})$ & Expected heterozygosity (SE) \\
\hline $1000 / 1000$ & $0.2400(0.0427)$ & $0.8857(0.0133)$ \\
$2000 / 2000$ & $0.1200(0.0325)$ & $0.9348(0.0066)$ \\
$2300 / 2300$ & $0.0600(0.0237)$ & $0.9396(0.0086)$ \\
$\mathbf{2 5 0 0 / 2 5 0 0}$ & $\mathbf{0 . 0 4 0 0 ( 0 . 0 1 9 6 )}$ & $\mathbf{0 . 9 4 2 6}(\mathbf{0 . 0 0 6 0})$ \\
$3000 / 3000$ & $0.0200(0.0140)$ & $0.9500(0.0066)$ \\
\hline
\end{tabular}

\section{Appendix 2.}

Detailed calculation of carrying capacity.

$$
\begin{aligned}
& 2.1 m+2.7 n=4,075 \\
& (m+n) /(2 m+n)=0.64 \\
& K=2 m+n
\end{aligned}
$$

Where $\mathrm{m}$ is the number of paired male individuals, $\mathrm{n}$ is the number of unpaired male individuals, and $K$ denotes the carrying capacity. These three equations produce a $K$ value of 2,695 . 